\title{
Temperature Measurement with Photodiode in Different Operating Modes
}

\author{
Dimcho Pulov \\ Department of Mechanical and Precision Engineering \\ Technical University of Gabrovo \\ Gabrovo, Bulgaria \\ pulov@mail.bg
}

\begin{abstract}
A method for contactless temperature measurement with photodetectors in different operating modes has been developed. It is based on the periodic change of the spectral sensitivity of the photodiode depending on the applied reverse voltage. This is accomplished by switching the photodiode within one measurement from photovoltaic to photoconductive mode. For this purpose, the scheme of the respective electronic key has been developed.
\end{abstract}

Keywords - contactless measuring of temperature, IR diapason, photovoltaic and photoconductive mode, spectral ratio pyrometer .

\section{INTRODUCTION}

Temperature is one of the main parameters of most modern production technologies. For this reason, its measurement and control is essential for the correct implementation of the respective technological process. This can be done both by traditional contact methods and by applying non-contact optical methods. The two main ways in which the method of optical pyrometry can be realized: energy and spectral ratio, are described in [4], [7], [10], [12], [14], [16]. For its part, the pyrometers of the spectral ratio can be realized in three variants: with two photodetectors, light-dividing device and narrow-band optical filters; with one tandem photodetector and with a matrix photodetector. It has been shown that in the pyrometers of the spectral ratio the uncertainty can be reduced when measuring the temperature, which arises as a result of:

a) Different distance to the objects and their different shape;

b) Different temperature of the environment;

c) Presence of side background objects in the field of view of the device;

\author{
Tsanko Karadzhov \\ Department of Mechanical and Precision Engineering \\ Technical University of Gabrovo \\ Gabrovo, Bulgaria \\ karadjov_s@abv.bg
}

d) Presence of nearby high temperature sources and reflection of their radiation to the device;

e) Obscurity of the radiation coefficient of the objects and its dependence on the temperature and wavelength, i.e. $\varepsilon=f(\lambda, T)$.

All the mentioned pyrometers of the spectral ratio have a significant drawback. They correctly measure the temperature of only gray objects for which $\varepsilon=$ const. If the objects are non-gray (selectively radiating), then for them the radiation coefficient will depend on the wavelength, i.e. $\varepsilon=f(\lambda)$. In this case, the ratio between the refractive indices in two narrow spectral ranges $\varepsilon_{\lambda 1} / \varepsilon_{\lambda_{2}}$ may change and lead to a measurement error. This error will be the greater the steeper the function $\varepsilon=f(\lambda)[1],[6]$. For some metals it can reach up to $10 \%$.

These disadvantages can be reduced with pyrometer with a single photodiode operating in series in different operating modes.

\section{EXPOSITION}

The goal of this publication is to develop a spectral ratio pyrometer using only one photodiode operating in two different modes to increase the accuracy and sensitivity of non-contact temperature measurement.

The set goal can be achieved by solving the following tasks:

- Development of a block functional scheme of the pyrometer;

- Development of a schematic electrical circuit diagram of the pyrometer;

- Development of the control program. 


\section{A. Essence of the method}

It is known that the photodiode can operate in two modes: photovoltaic and photoconductive. There is no external power supply in photovoltaic mode and the photodiode generates photo-EMF. The maximum voltage sensitivity in this mode is

$$
S_{U \max }=R_{0}\left[\frac{S_{i} I_{s}}{S_{i} \Phi_{\max }+I_{s}}\right]
$$

where: $S_{i}$ - sensitivity by current; $\Phi_{\max }$ - falling optical flux; $I_{s}$ - sum of the reverse drift currents of the non-core carriers; $R_{0}$ - resistance of $p-n$ junction.

Here

$$
R_{0}=k T / e I_{s}
$$

where: $e$ - electron charge; $k$ - Boltzmann's constant; $T$ temperature of $p-n$ junction.

At room temperature $k T / e=0.025 \mathrm{~V}$ and expression (1) is as follows:

$$
S_{U \max }=0.025\left[\frac{S_{i}}{S_{i} \Phi_{\max }+I_{s}}\right]
$$

In the photoconductive mode, a reverse voltage supply is connected in series with the photodiode. In this case, a reverse current flows through the photodiode, which is proportional to the optical flux falling on it. The voltage sensitivity in photoconductive mode is defined as

$$
S_{U}=\frac{d U_{n}}{d \Phi}=S_{i} R_{n}
$$

where: $U_{n}$ - voltage drop across the electrical load; $\Phi$ falling optical flux; $R_{n}$ - load resistance.

Therefore, the voltage sensitivity can be increased if the load resistance increases. In order for the photodiode to operate in linear mode, the maximum load resistance $R_{n \text { max }}$ must be related to the falling optical flux by the expression

$$
R_{n \max }=\frac{U_{p}}{I_{\Phi}+I_{s}}=\frac{U_{p}}{S_{i} \Phi+I_{s}}
$$

where: $U_{p}$ is the supply voltage; $I_{\Phi}$ is the photocurrent of non-core carriers (current in the dark).

Then the maximum voltage sensitivity in photoconductive mode is

$$
S_{u \max }=\frac{S_{i} U_{p}}{S_{i} \Phi+I_{s}}
$$

From expressions (1) and (2) the ratio between the sensitivities of the photodiode in photoconductive mode $S_{u \max F C}$ and in photovoltaic mode $S_{u \max F V}$ can be determined

$$
\frac{S_{u \max F C}}{S_{u \max F V}}=\frac{U_{p}}{k T / e}
$$

It is clear that the maximum sensitivity in photoconductive mode is higher than in photovoltaic mode.

Graphs of the spectral sensitivity of the Hamamatsu $S 2386$ photodiode $S_{F C}(\lambda)$ in photoconductive mode and $S_{F V}(\lambda)$ in photovoltaic mode are shown in Fig.1.

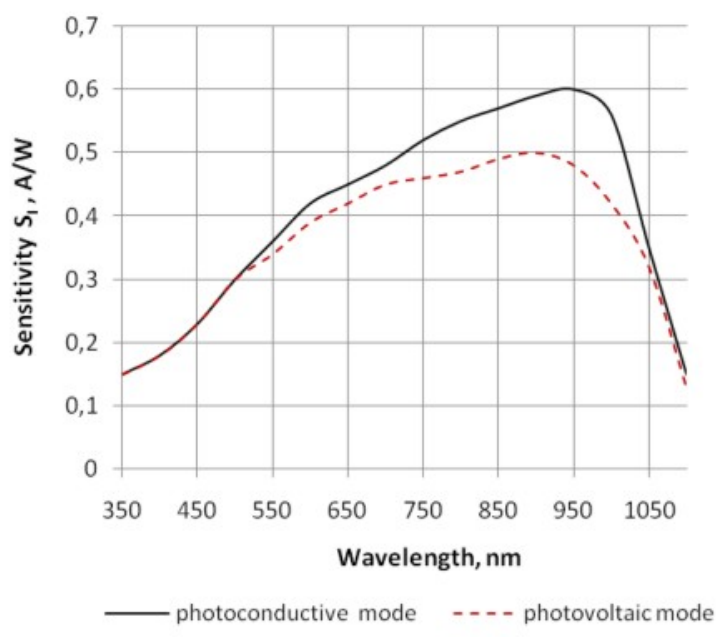

Fig. 1. Spectral sensitivity of the Hamamatsu S2386 photodiode in photoconductive and photovoltaic mode

The spectral sensitivity of the photodiode depends mainly on the properties of the semiconductor material from which it is made [8], [17]. As the reverse voltage increases (switching from photovoltaic to photoconductive mode) the volume charge, the potential barrier height and the $p-n$ junction width increase. All this leads to an increase in the collection coefficient of non-core charge carriers. As a consequence, there is an increase in the maximum of the spectral sensitivity of the photodiode and its displacement in the long-wavelength region [9], [16]. These properties of the photodiode can be used in a pyrometer in order to simplify its design and increase the accuracy and sensitivity of pyrometric measurements [11], [13].

The proposed method consists in the use of one photodiode and in the sequential switching within one measurement of its modes from photovoltaic to photoconductive, whereby its sensitivity and, consequently, the photocurrent flowing through it will be successively changed. In goal to minimize the 
methodological error, the operating spectral range will be relatively wide.

The electric current at the output of the photodiode is determined by the expression

$$
I(T)=A \int_{\Delta \lambda} M(\lambda, T) S(\lambda) d \lambda
$$

where: $M(\lambda, T)$ - the spectral exitance of the studied object; $S(\lambda)$ - the spectral sensitivity of the photodiode; $\Delta \lambda$ the operating spectral range of the pyrometer; $A$ coefficient depending on the parameters of the lens, the photodiode and the distance between them.

According to the proposed method, the output signal of the pyrometer $K_{I}$ will be the ratio of the individual signals received in the two modes of operation of the photodiode

$$
K_{I}(T)=\frac{I_{F C}}{I_{F V}}
$$

where: $I_{F C}$ - photocurrent in photoconductive mode;

$I_{F V}$ - photocurrent in photovoltaic mode.

The pyrometer is graduated according to a reference blackbody radiation source (bb). In this case, according to (3) and (4) it can be written

$$
K_{I}(T)=\frac{\int_{\Delta \lambda_{F C}} M_{b b}(\lambda, T) S_{F D}(\lambda) d \lambda}{\int_{\Delta \lambda_{F V}} M_{b b}(\lambda, T) S_{F V}(\lambda) d \lambda}
$$

where: $M_{b b}(\lambda, T)$ - spectral exitance of the reference.

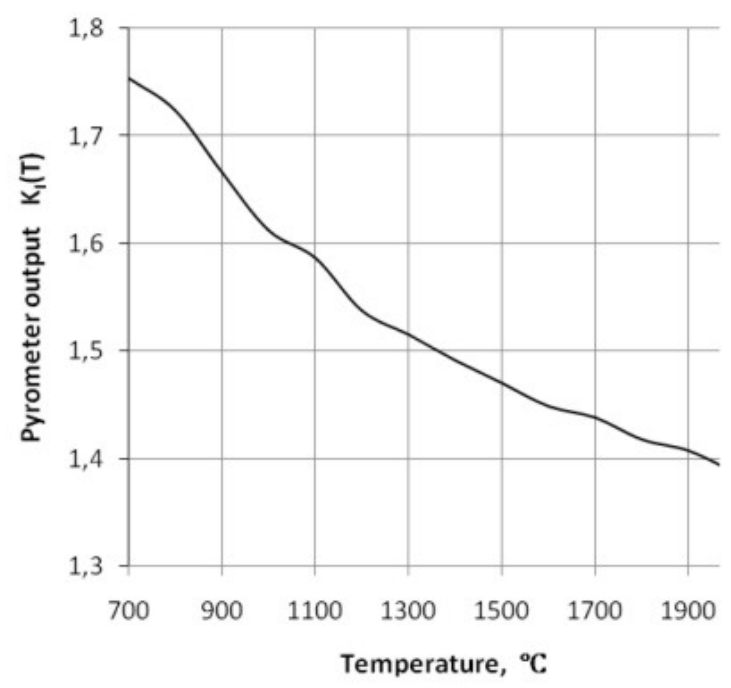

Fig. 2. The dependence $K_{I}(T)$
According to expression (5) the dependence $K_{I}(T)$ is drawn, which is shown in Fig. 2.

The total error in the pyrometer of the spectral ratio is determined by the instrumental error, the error of calibration of the pyrometer on a black body, the presence of parasitic reflections and the methodological error caused by the uncertainty of the emission coefficient. The methodological error has the greatest influence. It is shown in [2], [3], [5], [15] that when using wide spectral ranges and near the maxima of the spectral sensitivity of the two photodetectors, the methodological error decreases to $3.5 \%$. The instrumental error is comparable to that of spectral ratio pyrometers using two photodetectors and narrow spectral ranges, as well as tandem and matrix photodetectors.

\section{B. Block functional diagram of the pyrometer}

The block functional scheme of the pyrometer is shown in Fig. 3. The lens forms an image of the object under study on the photodiode (Hamamatsu S2386). A light filter is situated between the lens and the photodiode that absorbs the radiation in the visible range of the spectrum. The function of the light filter is to eliminate the influence of visible light on the measurement. FELO70 was selected as a filter from the Thorlabs catalog. This is a long-wave cutoff filter that transmits radiation with a wavelength of $\lambda>750 \mathrm{~nm}$. The scheme works in two cycles. In the first cycle, the electronic switch S, according to the instructions from the microcontroller, switches the anode of the photodiode to ground. In this case, the photodiode operates in photovoltaic mode. The output current from the photodiode is converted into voltage by an operational measuring amplifier OA. The voltage from the output of the amplifier is converted into digital form by a 10-bit ADC of the microcontroller and stored.

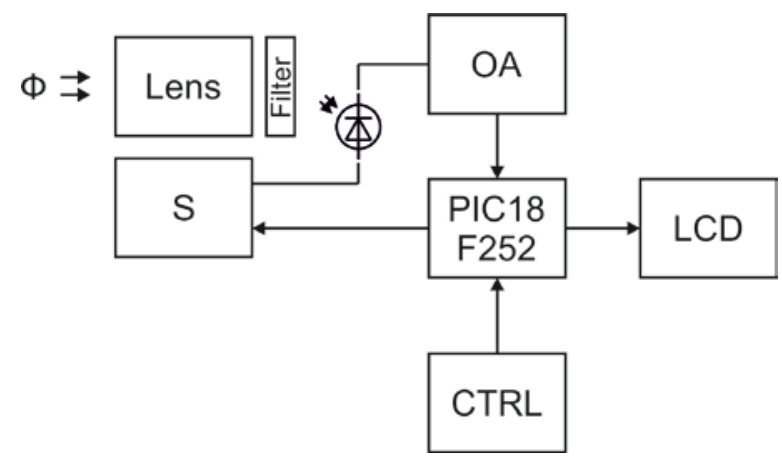

Fig. 3. Block chart of an optoelectronic device for measuring the power of laser radiation

In the second cycle, the electronic switch under the instruction of the microcontroller supplies a negative voltage of $-14 \mathrm{~V}$ to the anode of the photodiode. Then the photodiode operates in photoconductive mode. The output current from the photodiode is similarly converted to voltage, then digitally and stored. After the end of the second cycle, the microcontroller calculates the ratio of the output signals of the photodiode in the two operating 
modes. The information from the measured temperature is visualized on a two-line liquid alphanumeric indicator.

\section{Schematic electrical circuit diagram}

Fig. 4 shows the schematic electrical circuit of the device. An 8-bit PIC18F252 microcontroller with a builtin 10-bit ADC was used to control the operation of the entire device. The controller has a sufficient number of input-output ports, memory for measurement data and program memory, where the control program can be located. The threshold voltages that determine the measuring range are from $0 \mathrm{~V}$ to $5 \mathrm{~V}$. The number of digital levels in this range is 210 or 1024 levels. The step of increasing the voltage is determined by the formula:

$$
\Delta U=\frac{U_{C C}}{2^{10}}=\frac{5}{1024}=4.88 \mathrm{mV}
$$

he voltage from the operational amplifier is fed to the analog input RA2 / AN2. Two consecutive voltage measurements are made at this input and the photodiode is switched synchronously from photovoltaic mode to photoconductive mode via digital output RB7.

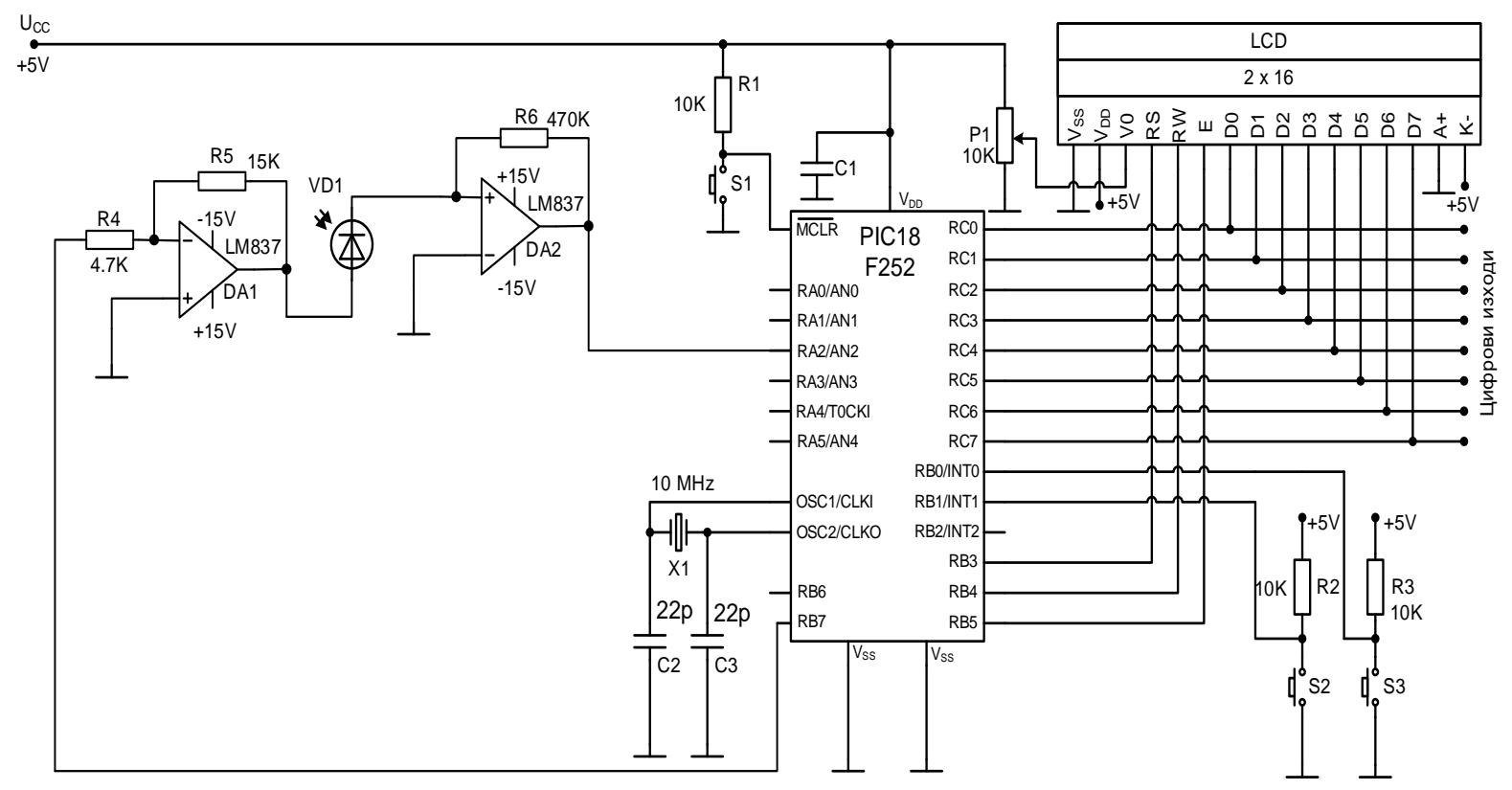

Fig. 4. Schematic electrical circuit diagram of the device

\section{Description of the control program}

First, the controller is initialized, constants and variables are defined and corresponding cells from the data memory are reserved for them. The next stage of the block chart is related to determining the $\mathrm{I} / \mathrm{O}$ ports to be used. Digital inputs, analog inputs and digital outputs are determined. PIC18F252 has 4 internal module timers. Only Timer 0 is used. It sets time lags in the program which are used to synchronize the operation of the microcontroller and the alphanumeric LCD. Next, an initialization of the display is performed. The master program is a continuous loop, which stops only when the voltage measurement from the ADC (analog-to-digital converter) has been completed. The processing of the result of each measurement takes place in a subroutine, which starts when an ADC interrupt occurs. At the beginning of the subroutine an analog input for the measurement is selected. The result of the previous measurement is then processed, which includes - hardware multiplication of the measurement value by a preset constant. The ratio $U_{1} / U_{2}$ between the voltages measured in the two modes is determined. The result is converted from hexadecimal to binary-decimal code. The second stage of the subroutine is related to outputting the measurement results on an LCD display. First, the output addresses are defined, it means - to determine in which position to display each digit from the result, including the decimal points. The values of the calculations are then displayed. Finally, a subsequent measurement is started in the subroutine and then returning to the master program takes place.

Connecting to the microcontroller and controlling the LCD display.

The display used is a two-line, alphanumeric, liquid crystal display, with 20 characters per line. It is a smart display with its own control scheme - LCD driver hd44780. The display has a total of 16 ports to enable power supply and connection to an external microcontroller. For controlling the LCD module a control bus is used comprising RS, R/W, E ports which are correspondingly connected to microcontroller ports RB3, RB4, RB5 initialized as digital outputs. The data bus of this type of display consists of 8 ports. The microcontroller communicates with the LCD module by means of the data bus. In this case a mode is selected for this communication to take place using 4-bit interface. The DB0, DB1, DB2, DB3, DB4, DB5, DB6, DB7 display ports are initialized as inputs and correspondingly connected to the 
microcontroller's RD0, RD1, RD2, RD3, RD4, RD5, RD6, RD7 ports which are initialized as outputs. At the beginning of the program, initialization of the display takes place, which selects the following:

a) 8-bit interface;

b) two-line mode;

c) character format $5 \times 8$ dots;

d) incrementing the address counter by one unit when in read/record mode.

\section{CONCLUSION}

A two-spectrum pyrometer using only one photodiode has been developed. The device can be used to measure temperature in a wide range from $700^{\circ} \mathrm{C}$ to $2000^{\circ} \mathrm{C}$. The spectral ratio is obtained by switching the photodiode from photovoltaic mode to photoconductive mode. The device operates in two wide spectral ranges and the corresponding two maxima of the spectral sensitivity of the photodetector are close. The use of the proposed scheme leads to an increase in the accuracy and sensitivity of the measurements.

\section{REFERENCES}

[1] A. G. Senkov, V. A. Firago, „Optimizaciya harakteristik pirometrov spektralnogo otnosheniya”, Vestn. BGU, Vol. 1, no. 3, 2008, pp. 38-44.

[2] A. G. Senkov and V. A. Firago, „Reduction of methodological errors in determining the temperature of metals by two-color pyrometers”, Journal of Engineering Physics and Thermophysics, Vol. 79, 2006, pp. 768-772.

[3] A. V. Frunze, „Metodicheskie pogreshnosti pirometrov I sposobi ih minimizacii”, Metrologiya, Vol. 7, 2012, pp.25-38.

[4] A. V. Frunze, „A numerical method of determining the spectralratio temperature”, Measurement Techniques, Vol. 53, 2010, pp. 664-667.

[5] A.V. Yakovlev, „Osobennosti primeneniya shirokopolosnih priemnikov izlucheniya v pirometrah spektralnogo otnosheniya”, Avtometriya., Vol. 4, 2004, pp. 44-49.
[6] D. Dichev, I. Zhelezarov, R. Dicheva, D. Diakov, H. Nikolova and G. Cvetanov, „Algorithm for estimation and correction of dynamic errors”, 30th International Scientific Symposium Metrology and Metrology Assurance. MMA 2020; Sozopol; Bulgaria, 2020, DOI: 10.1109/MMA49863.2020.9254261.

[7] M.S. Jadin and S. Taib, „Two-Color Pyrometer for Process Temperature Measurement During Machining”, Journal of lightwave technology, Vol. 34, no. 4, 2016, pp. 1380-1386: DOI: 10.1109/JLT.2015.2513158 .

[8] K. Chrzanowski, Non-Contact Thermometry. Measurement Errors, SPIE Polish Chapter, Warsaw, 2001.

[9] L. Lazov, N. Angelov, E. Teirumnieks and Erika Teirumnieka, Preliminary Numerical Analysis for the Role of Speed on to Laser Technological Processes, Proceedings of the 12th International Scientific and Practical Conference, Rezekne Latvia, , Vol. 3, 2019, pp. 137- 142, DOI: 10.17770/etr2019vol3.4154.

[10] Ts. Karadzhov, D. Pulov and N. Angelov, „Contactless measuring of temperature with differential photo receiver”, in 12th International Scientific and Practical Conference on Environment. Technology. Resources, Rezekne Academy of Technologies, Vol. 3, 2019, pp. 101-104 , DOI: 10.17770/etr2019vol3.4132.

[11] D. Dichev, H. Koev, T. Bakalova and P. Louda, „A Model of the Dynamic Error as a Measurement Result of Instruments Defining the Parameters of Moving Objects”, Measurement Science Review, Vol. 14, Issue 4, 2014, pp. 183-189, DOI: 10.2478/msr-2014-0025.

[12] A.N. Magunov, „Spektralnaya pirometriya: osobennosti, preimushchestva, ogranicheniya”, Fotonika, Vol. 1, 2012, pp. 4045.

[13] T. Fu, J. Liu, M. Duan and A. Zong, „Temperature measurements using multicolor pyrometry in thermal radiation heating environments”, Review of Scientific Instruments, Vol. 85, Issue 4, 044901, 2014, DOI: 10.1063/1.4870252.

[14] D. Ya. Svet, Opticheskie metodi izmereniya istinnih temperatur, Moskva, Nauka, 1982.

[15] A. Araujo, Multi-spectral pyrometry - a review, „Measurement Science and Technology”, Vol. 28, Issue 8, 082002, 2017, DOI: 10.1088/1361-6501/aa7b4b.

[16] D. B. Ponomarev and V. A. Zakharenko, Silicon photodiode as the two-color detector, Journal of Physics: Conference Series , Vol. 643, Issue 1, 2015, DOI: 10.1088/1742-6596/643/1/012030.

[17] G. G. Ischanin and V. P. Chelibanov, Priemniki opticheskogo izlucheniya, Sankt Peterburg, Lan, 2014. 